\title{
A NOTE ON THE STEADY-STATE RESPONSE OF AN ELASTIC HALF-SPACE
}

\author{
By N. C. TSAI
}

\section{ABSTRACT}

\begin{abstract}
With reference to the influence of local geology on earthquake ground motions, a more complete analytical formulation is made of the well-known problem of a horizontally stratified, linearly-elastic half-space subjected to vertically traveling, sinusoidal, plane waves. A more general interpretation of a result of Kanai is given, and a recursion formula is derived for computing amplification spectra. Some special properties of the system are pointed out and numerical examples are given.
\end{abstract}

\section{INTRODUCTION}

The influence of local geology on the shaking of the ground is of much interest for its engineering implications. In Mexico City, for example, the spectra of the recorded earthquake motions on the surface of the old lake bed have a prominent peak at a period of 2.5 seconds which is interpreted as showing the influence of local geology. In engineering considerations local geology is usually taken to mean the alluvial layers underlying a site as these would have the most pronounced influence on the seismic waves. An analysis of this problem, taking into account the propagation of the seismic waves from their origin to the site and taking into account the true physical and geometrical properties of the soil deposits and the underlying rock, would be exceedingly difficult. A simplified form of this problem, originally studied by Kanai and Sezawa, considers a set of horizontal, elastic layers of uniform thickness overlying an elastic half-space. The layers are assumed to be excited into motion by a vertically traveling, plane wave which passes from the half-space into the layers, and after reflections and refractions the waves pass back into the half-space. The problem is thus reduced to one-dimensional wave propagation, either transient or steady-state. Although this idealized system differs from the conditions found in nature it is thought that its behavior can provide some insight into the behavior of real systems. In this paper the problem is given analytical formulation which incorporates the work of previous investigations appearing in various scattered publications.

By computing the steady-state response of a layered system to a sinusoidal excitation the frequency-selective property of the system can be clearly exhibited by means of an amplification spectrum which shows how the system amplifies some frequency components and suppresses others. Such studies have been made by Sezawa and Kanai (1930, 1932, 1935a, 1935b), Kanai (1952, 1953), Takahasi (1955), Matthiesen, Duke, Leeds, and Fraser (1964), and Herrera and Rosenblueth (1965). Haskell $(1953 ; 1962)$ has formulated in terms of transfer matrix the more general problem of a layered system excited by body waves that are incident obliquely rather than vertically. Kanai has treated viscous (Voigt) layered systems with one to three layers. Takahasi developed a graphical technique for finding the response of an N-layered nonviscous system. Matthiesen $e t a l$. derived the solution for a viscous (Voigt) N-layered system and the results were applied to study the site characteristics of strongmotion earthquake stations in southern California. Herrera and Rosenblueth derived 
the solution for a viscous $\mathrm{N}$-layered system in matrix form. Some conclusions of engineering significance were deduced from the steady-state analysis by Kanai:

(1) A nonviscous system with one layer only has a prominent resonant response while a multi-layered system has less prominent resonant responses.

(2) During earthquakes, there is a dominant period associated with the ground layers. This period is proportional to the total thickness of the layers.

(3) Due to the deformability of the base-rock foundation of the half-space, a portion of wave energy is transmitted back into the foundation. This energy loss is called "geometrical dissipation," (Kanai, 1963, 1964) and, consequently, the resonant response is bounded even if the system is nonviscous.

In this paper, there is re-derived the steady-state response of a linearly viscoelastic layered system in the form of a recursion formula from which several properties of the amplification spectrum can be deduced for a nonviscous system having its stiffnesses increasing with depth. From these conclusions, a more general interpretation can be given to Kanai's first conclusion. Numerical examples are presented to demonstrate the effect of layer parameters on the amplification spectrum, and a tentative conclusion is drawn for viscoelastic systems having gradually increasing stiffnesses and having fixed properties for the top layer and the foundation.

The following analysis is made for incident $S$ waves, but the results can readily be converted to the case of $P$ waves by replacing the corresponding elastic constant and the corresponding viscosity constant.

It should be noted that a more practical approach involves the analysis of transient excitations of more heavily damped layered systems, but this is better done by other methods (Tsai, 1969). Soils are not necessarily uniformly layered or linearly elastic, and all seismic waves are not planar and vertically traveling, so that the real problem is much more complicated than currently used methods of analysis indicate.

\section{Mathematical Analysis}

The linear viscoelastic models most commonly used to describe viscous dissipation are the Maxwell model, the Voigt model, and the standard linear model. Denoting the shear stress and the shear strain by $\sigma$ and $\epsilon$ respectively, the steady-state response is $\epsilon=e^{i \omega t}$ and $\sigma=\mu(\omega) e^{i \omega t}$, and a general stress-strain law can be written for the models as follows (Tsai, 1969):

$$
\sigma=\mu(\omega)
$$

For the Maxwell model with a "relaxation time" constant $\beta$,

$$
\mu(\omega)=\mu_{0} \frac{i \omega \beta}{1+i \omega \beta}
$$

where $\mu_{0}$ is the shearing modulus in the absence of viscosity. For the Voigt model and the standard linear model,

$$
\mu(\omega)=\frac{1+i \omega \tau}{1+\frac{i \omega \tau}{1+r}}
$$

where $\tau$ is the retardation-time constant, and $r$ is a nondimensional parameter, equal 
to $\infty$ for the Voigt model. By definition, the complex shear wave velocity is

$$
c(\omega)=\sqrt{\mu(\omega) / \rho}
$$

which is equal to $\sqrt{\mu_{0} / \rho}$ for a nonviscous material, $\rho$ being the density.

Consider a horizontally stratified $N$-layered linearly elastic system with each layer being homogeneous and isotropic, and obeying the general stress-strain law, equation 1 , for steady-state deformation. The layered system is shown in Figure 1 with the top layer indexed 1 and the half-space $N+1$. A set of $N$ coordinates, $z_{j}, j=1,2, \cdots, N$,

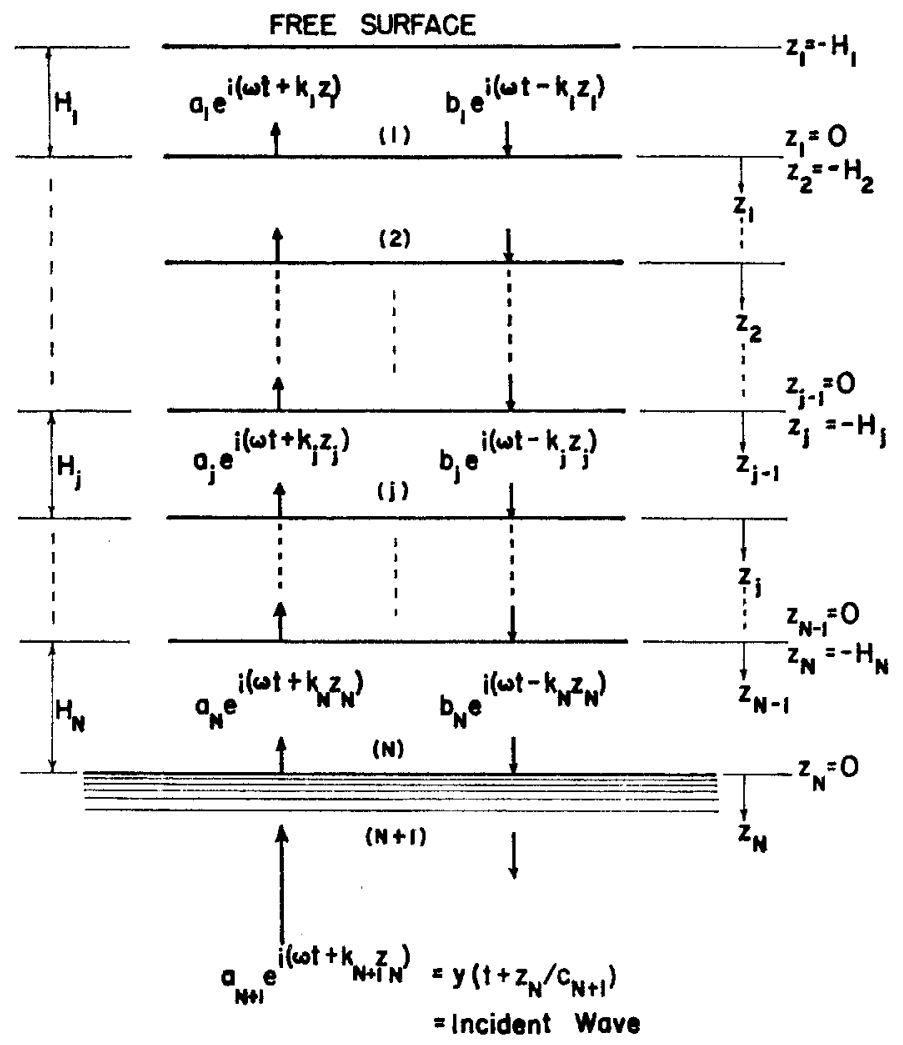

Fra. 1. An $N$-layer system under steady-state excitation

is also defined as shown. The vertically incident plane $S$ wave

$$
y\left(t+z_{N} / c_{N+1}\right)=a_{N+1} e^{i \omega\left(t+z_{N} / c_{N+1}\right)}
$$

with $a_{N+1}$ being a known amplitude, generates a response, $u_{j}\left(z_{j}, t\right)$, in the $j$ th layer described by the one-dimensional wave equation

$$
c_{j}^{2}(\omega) \frac{\partial^{2} u_{j}\left(z_{j}, t\right)}{\partial z_{j}^{2}}=\frac{\partial^{2} u_{j}\left(z_{j}, t\right)}{\partial t^{2}} \quad j=1,2, \cdots, N
$$

for $z_{j}=-H_{j}$ to $z_{j}=0$. The solution of equation (4) can be written as the sum of upward traveling wave and a downward traveling one

$$
u_{j}\left(z_{j}, t\right)=a_{j} e^{i\left(\omega t+k_{j} z_{j}\right)}+b_{j} e^{i\left(\omega t-k_{j} z_{j}\right)}
$$


in which $k_{j}=\omega / c_{j}(\omega)$ is the wave number. The amplitudes $a_{j}$ and $b_{j}$ are determined by applying the proper boundary conditions at each of the interfaces. At the free surface $z_{1}=-H_{1}$, the stress-free condition yields

$$
b_{1}=a_{1} e^{-2 i s_{1}}
$$

where it is defined that

$$
s_{j}=k_{j} H_{j} \quad j=1,2, \cdots, N .
$$

The conditions at the interfaces defined by $z_{j-1}=0$ and $z_{j}=-H_{j}$ are that the displacements are continuous, which gives

$$
a_{j-1}+b_{j-1}=a_{j} e^{-i s_{j}}+b_{j} e^{i s_{j}} .
$$

and that the shearing stresses are continuous, which gives

$$
\alpha_{j-1}\left(a_{j-1}-b_{j-1}\right)=a_{j} e^{-i s_{j}}-b_{j} e^{i s_{j}}
$$

In equation (9) $\alpha_{j-1}$ is the impedance ratio between the $(j-1)^{\text {th }}$ and $j^{\text {th }}$ layer and is equal to $\rho_{j-1} c_{j-1} / \rho_{j} c_{j}$.

From equations (8) and (9), $a_{j}$ and $b_{j}$ can be expressed in terms of $a_{j-1}$ and $b_{j-1}$ by

$$
\begin{aligned}
\left\{\begin{array}{l}
a_{j} \\
b_{j}
\end{array}\right\} & =\frac{1}{2}\left[\begin{array}{cc}
\left(1+\alpha_{j-1}\right) e^{i s_{j}} & \left(1-\alpha_{j-1}\right) e^{i \varepsilon_{j}} \\
\left(1-\alpha_{j-1}\right) e^{-i s_{j}} & \left(1+\alpha_{j-1}\right) e^{-i s_{j}}
\end{array}\right]\left\{\begin{array}{l}
a_{j-1} \\
b_{j-1}
\end{array}\right\} \\
& =[S]_{j}[T]_{j-1}\left\{\begin{array}{l}
a_{j-1} \\
b_{j-1}
\end{array}\right\} \quad j=2, \cdots, N
\end{aligned}
$$

where

$$
[S]_{j}=\left[\begin{array}{ll}
e^{i s_{j}} & \\
& e^{-i s_{j}}
\end{array}\right] \quad[T]_{j}=\frac{1}{2}\left[\begin{array}{ll}
1+\alpha_{j} & 1-\alpha_{j} \\
1-\alpha_{j} & 1+\alpha_{j}
\end{array}\right] .
$$

At the lowest interface, where $z_{N}=0$, we have

$$
\left\{\begin{array}{l}
a_{N+1} \\
b_{N+1}
\end{array}\right\}=[T]_{N}\left\{\begin{array}{l}
a_{N} \\
b_{N}
\end{array}\right\} \text {. }
$$

Upon repeated use of equation (10), $a_{j}$ and $b_{j}$ can be expressed in terms of $a_{1}$ and $b_{1}$, and, by using equation (6), equation (10) becomes

$$
\left\{\begin{array}{l}
a_{j} \\
b_{j}
\end{array}\right\}=a_{1} e^{-i s_{1}}\left([S]_{j}[T]_{j-1}\right) \cdots\left([S]_{2}[T]_{1}\right)[S]_{1}\left\{\begin{array}{l}
1 \\
1
\end{array}\right\} .
$$

For convenience the last equation can be written

$$
\left\{\begin{array}{l}
a_{j} \\
b_{j}
\end{array}\right\}=a_{1} e^{-i s_{1}}[S]_{j}\left\{\begin{array}{l}
\mathrm{Re}_{j}+i \operatorname{Im}_{j} \\
\operatorname{Re}_{j}-i \mathrm{Im}_{j}
\end{array}\right\} .
$$


It can be shown by induction that $R e_{j}$ and $I m_{j}$ are given by the following recursion formulas

$$
\begin{aligned}
& \operatorname{Re}_{j}=\operatorname{Re}_{j-1} \cos s_{j-1}-\operatorname{Im}_{j-1} \sin s_{j-1} \\
& \operatorname{Im}_{j}=\alpha_{j-1}\left(\operatorname{Re}_{j-1} \sin s_{j-1}+\operatorname{Im}_{j-1} \cos s_{j-1}\right)
\end{aligned}
$$

starting with

$$
\operatorname{Re}_{1}=1, \quad \operatorname{Im}_{1}=0
$$

Both $s_{j}$ and $\alpha_{j}$ are real, and hence $\operatorname{Re}_{j}$ and $\operatorname{Im}_{j}$ are real, only if the layers are nonviscous. For viscoelastic systems, $\mathrm{Re}_{j}$ and $\mathrm{Im}_{j}$ will be complex and their explicit expansions were derived by Tsai (1969).

Substituting equation 13 for $j=N$ into equation (12) gives

$$
a_{1}=\frac{e^{i s_{1}}}{\operatorname{Re}_{N+1}+i \operatorname{Im}_{N+1}} a_{N+1}
$$

Hence, $a_{j}$ and $b_{j}$ are given by equations (13) as

$$
\left\{\begin{array}{l}
a_{j} \\
b_{j}
\end{array}\right\}=\frac{a_{N+1}}{\operatorname{Re}_{N+1}+i \operatorname{Im}_{N+1}}[S]_{j}\left\{\begin{array}{l}
\operatorname{Re}_{j}+i \operatorname{Im}_{j} \\
\operatorname{Re}_{j}-i \operatorname{Im}_{j}
\end{array}\right\}
$$

and the response at the surface of the layered system will be

$$
u_{1}\left(-H_{1}, t\right)=\frac{2 a_{N+1}}{\sqrt{\operatorname{Re}_{N+1}^{2}+\operatorname{Im}_{N+1}^{2}}} e^{i\left(\omega t-\Phi_{N+1}\right)}
$$

with

$$
\Phi_{N+1}(\omega)=\tan ^{-1} \frac{\operatorname{Im}_{N+1}}{\operatorname{Re}_{N+1}}
$$

We define the following ratio

$$
\begin{aligned}
\operatorname{AMP}(\omega) & =\frac{\left|u_{1}\left(-H_{1}, t\right)\right|}{|2 y(t)|}=\frac{\left|u_{1}\left(-H_{1}, t\right)\right|}{2 a_{N+1}} \\
& =\frac{1}{\sqrt{\operatorname{Re}_{N+1}^{2}+\operatorname{Im}_{N+1}^{2}}}
\end{aligned}
$$

where $y(t)$ is the wave form of the incident wave. The plot of $\operatorname{AMP}(\omega)$ as a function of the frequency is the amplification spectrum for the surface response with respect to $2 y(t)$. Observe that the double amplitude, $2 a_{N+1}$, has been used in the definition of $\operatorname{AMP}(\omega)$ because $2 a_{N+1}$ would, according to equation (18), be the amplitude of the surface motion of the half-space foundation if there were no superposed layers. By defining

$$
G_{j}(\omega)=\sqrt{\operatorname{Re}_{j}^{2}+\operatorname{Im}_{j}^{2}}
$$


and

$$
\Phi_{j}(\omega)=\tan ^{-1} \frac{\operatorname{Im}_{j}}{\operatorname{Re}_{j}}
$$

the response of the $j^{\text {th }}$ layer is

$$
u_{j}\left(z_{j}, t\right)=2 a_{N+1} \operatorname{AMP}(\omega) G_{j}(\omega) \cos \left[k_{j}\left(H_{j}+z_{j}\right)+\Phi_{j}\right] e^{i\left(\omega t-\Phi_{N+1}\right)} .
$$

In the case of viscoelastic layered systems, AMP, $\Phi_{N+1}, G_{j}$, and $\Phi_{j}$ are complex because $\operatorname{Re}_{j}$ and $\operatorname{Im}_{j}$ are complex, and the explicit expansions for AMP and $u_{j}$ are given by Tsai (1969).

Because the infinite half-space participates in the motion, the system does not have mode shapes and natural frequencies in the classical sense. However, a characteristic frequency of a nonviscous layered system can be defined as follows. Let $\omega_{n}$ satisfy the relation

$$
\operatorname{Re}_{N+1}\left(\omega_{n}\right)=0 \quad n=1,2, \cdots
$$

We then have

$$
\begin{aligned}
A M P\left(\omega_{n}\right) & =\frac{1}{\left|\operatorname{Im}_{N+1}\left(\omega_{n}\right)\right|} \\
& =\frac{1}{\alpha_{N}\left|\operatorname{Re}_{N} \sin s_{N}+\operatorname{Im}_{N} \cos s_{N}\right| \omega=\omega_{n}}
\end{aligned}
$$

$\operatorname{AMP}\left(\omega_{n}\right)$ is seen to approach infinity as $\alpha_{N}$ approaches zero. In the limiting case that $\alpha_{N}=0$, the foundation is rigid and the system will have resonant response with infinite amplitude at $\omega_{n}$. Hence, equation (22) defines the characteristic frequencies, $\omega_{n}$, of a nonviscous system which are simply the natural frequencies of the system with a rigid foundation. Also, equation (22) indicates that the value of $\omega_{n}$, is independent of the property of the half-space foundation. In most realistic problems, the foundation would be more or less deformable so that $\alpha_{n}$ will be different from zero and $\operatorname{AMP}\left(\omega_{n}\right)$ will always be finite. This results from the fact that a certain amount of energy is lost from the layers by being transmitted back into the deformable foundation. Such an interpretation, first emphasized by Kanai $(1963,1964)$ is called the "geometric dissipation" of wave energy.

\section{Properties of AMP $(\omega)$}

A nonviscous layered system whose stiffnesses increase with depth, i.e., $0<\alpha_{j} \leqq 1$ for $j=1, \cdots, N$, is of most practical interest, and several important assertions concerning the nature of the amplification spectrum of such a system can be derived as follows:

Property $(A) \operatorname{AMP}(\omega)$ is always finite and is greater than or equal to 1 .

We first prove that $\mathrm{Re}_{j}$ and $\mathrm{Im}_{j}$ never vanish simultaneously. That this is true is obvious by inspection for the cases of $j=1$ and 2 ; for $j \geqq 3$, equation (20) gives

$$
\begin{aligned}
\operatorname{Re}_{j}^{2}+\operatorname{Im}_{j}^{2} / \alpha_{j-1}^{2} & =\left(\operatorname{Re}_{j-1} \cos s_{j-1}-\operatorname{Im}_{j-1} \sin s_{j-1}\right)^{2} \\
& +\left(\operatorname{Re}_{j-1} \sin s_{j-1}+\operatorname{Im}_{j-1} \cos s_{j-1}\right)^{2}=\operatorname{Re}_{j-1}^{2}+\operatorname{Im}_{j-1}^{2} .
\end{aligned}
$$


Rearranging the right-hand side, equation (23) becomes

$$
\operatorname{Re}_{j}^{2}+\operatorname{Im}_{j}^{2} / \alpha_{j-1}^{2}=\left(1-\alpha_{j-2}^{2}\right) \operatorname{Re}_{j-1}^{2}+\alpha_{j-2}^{2}\left(\operatorname{Re}_{j-1}^{2}+\operatorname{Im}_{j-1}^{2} / \alpha_{j-2}^{2}\right) .
$$

Upon repeated application of the above recursive relation, we finally arrive at

$$
\begin{aligned}
\operatorname{Re}_{j}{ }^{2}+\operatorname{Im}_{j}{ }^{2} / \alpha_{j-1}^{2} \prod_{k=1}^{j-2} \alpha_{k}{ }^{2}+\sum_{k=2}^{j-1} \operatorname{Re}_{k}{ }^{2}\left(1-\alpha_{k}{ }^{2}\right)\left(\prod_{i=k}^{j-2} \alpha_{i}{ }^{2}\right) & \\
j & =3, \cdots, N+1 .
\end{aligned}
$$

The right-hand side of equation (24) is obviously greater than zero, and hence $\operatorname{Re}_{j}$ and $\mathrm{Im}_{j}$ cannot vanish simultaneously.

On the other hand, adding $\operatorname{Im}_{j}^{2}\left(1-1 / \alpha_{j-1}^{2}\right)$ to both sides of equation (23) gives

$$
\operatorname{Re}_{j}^{2}+\operatorname{Im}_{j}^{2}=\operatorname{Re}_{j-1}^{2}+\operatorname{Im}_{j-1}^{2}-\operatorname{Im}_{j}^{2}\left(1-\alpha_{j-1}^{2}\right) / \alpha_{j-1}^{2}
$$

Applying this recursive relation repeatedly gives

$$
\operatorname{Re}_{j}^{2}+\operatorname{Im}_{j}^{2}=1-\sum_{k=1}^{j-1} \operatorname{Im}_{k+1}\left(1-\alpha_{k}^{2}\right) / \alpha_{k}^{2}
$$

Since $\alpha_{k}{ }^{2} \leqq 1$ for $k=1,2, \cdots, N$, the sum in equation (25) will always be positive. Equations (24) and (25) lead to the following inequality

$$
0<\operatorname{Re}_{j}^{2}+\operatorname{Im}_{j}^{2} \leqq 1 \text {. }
$$

Setting $j=N+1$, it immediately follows from the definition of AMP $(\omega)$ that

$$
\infty>\operatorname{AMP}(\omega) \geqq 1 \text {. }
$$

Property $(B)$. The value of AMP $\left(\omega_{n}\right)$ has an upper bound equal to $1 /\left(\alpha_{1} \cdot \alpha_{2} \cdots \alpha_{N}\right)$ and a lower bound $1 / \alpha_{N}$.

Making use of equation (23) for $j=N+1$ gives

$$
\operatorname{Re}_{N+1}^{2}+\operatorname{Im}_{N+1}^{2} / \alpha_{N}^{2}=\operatorname{Re}_{N}^{2}+\operatorname{Im}_{N}^{2}
$$

Since $\operatorname{Re}_{N+1}\left(\omega_{n}\right)$ vanishes (equation (23)), it follows that

$$
\operatorname{Im}_{N+1}^{2}\left(\omega_{n}\right)=\alpha_{N}^{2}\left[\operatorname{Re}_{N}^{2}\left(\omega_{n}\right)+\operatorname{Im}_{N}^{2}\left(\omega_{n}\right)\right]
$$

Making use of equation (25) for $j=N$,

$$
\begin{aligned}
\operatorname{Im}_{N+1}^{2}\left(\omega_{n}\right) & =\alpha_{N}{ }^{2}\left[1-\sum_{k=1}^{N-1} \operatorname{Im}_{k+1}^{2}\left(\omega_{n}\right)\left(1-\alpha_{k}^{2}\right) / \alpha_{k}{ }^{2}\right] \\
& \leqq \alpha_{N}{ }^{2}
\end{aligned}
$$

or

$$
\left|\operatorname{IM}_{N+1}\left(\omega_{n}\right)\right| \leqq \alpha_{N}
$$


On the other hand, setting $j=N+1$ in equation (24) gives

$$
\begin{aligned}
\operatorname{Im}_{N+1}^{2}\left(\omega_{n}\right) / \alpha_{N}{ }^{2} & =\prod_{k=1}^{N-1} \alpha_{k}^{2}+\sum_{k=2}^{N} \operatorname{Re}_{k}^{2}\left(\omega_{n}\right)\left(1-\alpha_{k-1}^{2}\right)\left(\prod_{i=k}^{N-1} \alpha_{i}^{2}\right) \\
& \geqq \prod_{k=1}^{N-1} \alpha_{k}{ }^{2}
\end{aligned}
$$

or simply

$$
\left|\operatorname{Im}_{N+1}\left(\omega_{n}\right)\right| \geqq \alpha_{1} \alpha_{2} \cdots \alpha_{N}
$$

Equation (28) and the last equation together define the bounds for $\operatorname{AMP}\left(\omega_{n}\right)$ as

$$
\frac{1}{\alpha_{N}} \leqq A M P\left(\omega_{n}\right) \leqq \frac{1}{\alpha_{1} \alpha_{2} \cdots \alpha_{N}}
$$

Property $(C)$ AMP $\left(\omega_{n}\right)$ assumes the minimum value given by property $(B)$ if the characteristic frequency satisfies

$$
\sin s_{j}\left(\omega_{n}\right)=\cos \varepsilon_{N}\left(\omega_{n}\right)=0 \quad j=1, \cdots, N-1
$$

and the maximum value if

$$
\cos s_{1}\left(\omega_{n}\right)=\sin s_{j}\left(\omega_{n}\right)=0 \quad j=2, \cdots, N .
$$

For a single-layered system $(N=1), \operatorname{AMP}\left(\omega_{n}\right)$ equals a constant, $1 / \alpha_{1}$, which is both the maximum and minimum itself. For $N \geqq 2$, equation (22) gives

$$
\operatorname{Re}_{N+1}\left(\omega_{n}\right)=\operatorname{Re}_{N}\left(\omega_{n}\right) \cos s_{N}\left(\omega_{n}\right)-\operatorname{Im}_{N}\left(\omega_{n}\right) \sin _{N}\left(\omega_{n}\right)=0
$$

The characteristic frequency, $\omega_{n}$, in general does not make $\operatorname{AMP}\left(\omega_{n}\right)$ a maximum or minimum unless some particular conditions are met by the layer parameters.

(1) From equation (27), there will be a minimum AMP $\left(\omega_{n}\right)$ equal to $1 / \alpha_{N}$ only if $\omega_{n}$ satisfies

$$
\left|\operatorname{Im}_{N+1}\left(\omega_{n}\right)\right|=\alpha_{N}
$$

The last condition implies that, in equation (27), the following condition should be fulfilled

$$
\operatorname{Im}_{j}\left(\omega_{n}\right)=0 \quad j=2, \cdots N
$$

which in turn implies that

$$
\sin s_{j}\left(\omega_{n}\right)=0 \quad j=1, \cdots N-1
$$

In addition, substituting equation (34) for $j=N$ into equation (33) gives

$$
\cos s_{N}\left(\omega_{n}\right)=0
$$


because, by property $(\mathrm{A}), \mathrm{Re}_{N}\left(\omega_{n}\right)$ cannot vanish. The last condition, together with equation (35), yields equation (31) - the condition under which the minimum value of AMP $\left(\omega_{n}\right)$ occurs.

(2) From equation (29), the maximum of AMP $\left(\omega_{n}\right)$ will occur if $\omega_{n}$ satisfies

$$
\left|\operatorname{IM}_{N+1}\left(\omega_{n}\right)\right|=\alpha_{1} \alpha_{2} \cdots \alpha_{N}
$$

or

$$
\operatorname{Re}_{j}\left(\omega_{n}\right)=0 \quad j=2, \cdots, N
$$

which in turn implies that, from the recursion formula for $\mathrm{Re}_{j}$,

$$
\cos s_{1}\left(\omega_{n}\right)=0 \text { and } \sin s_{j}\left(\omega_{n}\right)=0 \quad j=2, \cdots, N-1
$$

Substituting equation (36) for $j=N$ into equation (33) gives the additional condition

$$
\sin s_{N}\left(\omega_{n}\right)=0
$$

The last condition together with equation (37) constitute the condition, equation (32), under which the maximum of AMP $(\omega)$ occurs.

Property $(D)$. A layer is said to be transparent with respect to a particular frequency $\Omega$ if the thickness of said layer is equal to an integer multiple of the half-wave length. This property is true for any nonviscous layered system.

First of all, by transparent layer we mean that at a particular frequency $\Omega$ the amplification spectrum is completely independent of the parameters of the layer. In other words, AMP $(\Omega)$ can be computed as if this layer is absent. Let us consider the $j^{\text {th }}$ layer and suppose its thickness to be an integer multiple of the half-wave length at $\Omega$, namely,

$$
H_{j}=\frac{m}{2} \lambda_{j}(\Omega) \quad m=1,2, \cdots
$$

where $\lambda_{j}$ is the wave length equal to $2 \pi / k_{j}(\Omega)$. Since equation (38) implies that

$$
s_{j}(\Omega)=k_{j}(\Omega) H_{j}=m_{\pi}
$$

we have

$$
\sin s_{j}(\Omega)=0, \quad \cos s_{j}(\Omega)=(-1)^{m}
$$

Substituting the last equation into the recursion formula for $\mathrm{Re}_{j+1}$ gives

$$
\begin{aligned}
\operatorname{Re}_{j+1}(\Omega) & =(-1)^{m} \operatorname{Re}_{j}(\Omega) \\
& =(-1)^{m}\left[\operatorname{Re}_{j-1} \cos s_{j-1}-\operatorname{Im}_{j-1} \sin s_{j-1}\right]_{\omega=\Omega}
\end{aligned}
$$

Similarly we can obtain

$$
\operatorname{Im}_{j+1}(\Omega)=(-1)^{m} \frac{\rho_{j-1} c_{j-1}}{\rho_{j+1} c_{j+1}}\left[\operatorname{Re}_{j-1} \sin s_{j-1}+\operatorname{Im}_{j-1} \cos s_{j-1}\right]_{\omega=\Omega}
$$


Both $\operatorname{Re}_{j+1}(\Omega)$ and $\operatorname{Im}_{j+1}(\Omega)$ are obviously independent of the parameters of the $j^{\text {th }}$ layer, which implies that $\operatorname{Re}_{N+1}(\Omega)$ and $\operatorname{Im}_{N+1}(\Omega)$ can be computed as if the $j^{\text {th }}$ layer is absent. Hence, property $(D)$ is established.

As already mentioned, Kanai had drawn the conclusion that a single-layer system has prominent resonant amplification while, on the other hand, resonant amplification of a multi-layer system is less prominent. He attributed this behavior of multilayer systems to the complicated interference of the waves during reflection and transmission across the layer interfaces. Intuitively, since both systems are physically similar and differ from each other only in the layer parameters both would be ex-

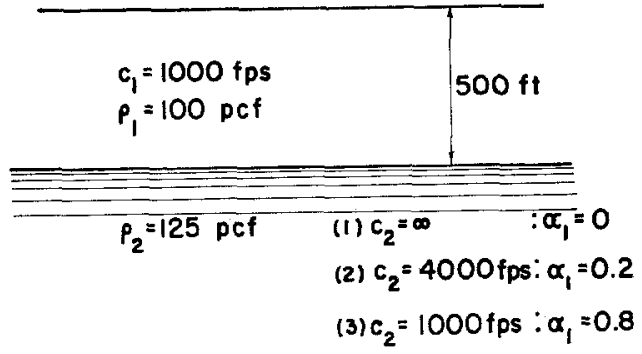

(a) THE SINGLE-LAYER SYSTEM ( $N=1)$

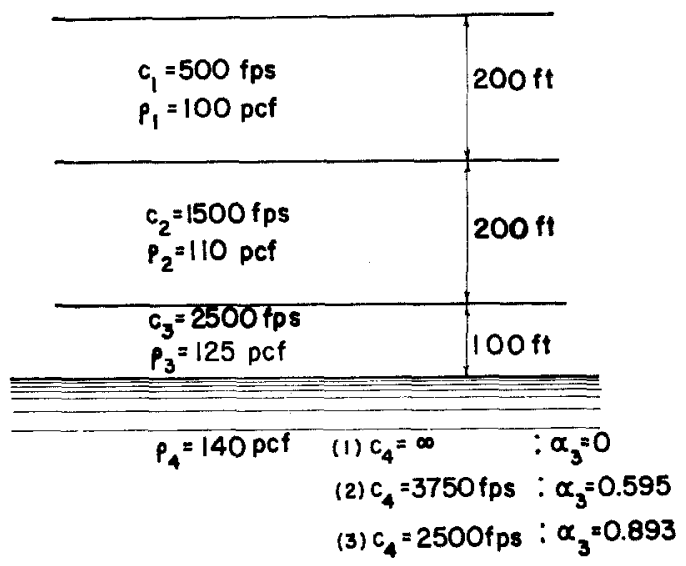

(b) THE TRIPLE - LAYER SYSTEM $N=3$ )

Fra. 2. Layered systems for demonstrating the effect of $\alpha_{N}$.

pected to have resonant responses of comparable prominence, so that Kanai's conclusion seems inconsistent. The following explanation is given for this apparent inconsistency.

Theoretically, property $(B)$ indicates that a nonviscous single-layer system has a regular resonant amplification, AMP $\left(\omega_{n}\right)$, equal to $1 / \alpha_{1}$. The value of $\alpha_{1}$ depends on the parameters of the system given and, therefore, need not necessarily be small. Hence, the resonant amplifications of a single-layer system need not be prominent. On the other hand, let us consider a nonviscous multi-layer system that has layer stiffnesses increasing with depth. The values of AMP $\left(\omega_{n}\right)$ are no longer regular, and 
the range of possible variations of AMP $\left(\omega_{n}\right)$ is defined by the bounds given in property $(B)$. In general, some resonant amplifications may be prominent while the others are suppressed. Yet, in spite of these possible variations, all the resonant amplifications will be prominent if $\alpha_{N}$ is sufficiently small. In short, it can be seen that Kanai's conclusion does not hold in general but is valid only for those special systems he analyzed. For illustration, a single-layer system and a triple-layer system, arbitrarily chosen, are shown in Figures 2(a) and 2(b) respectively. For each system, amplification spectra have been computed for three different values of $\alpha_{N}$ obtained by varying the wave velocity of the foundation. The results are shown in Figure 3, which indicate that $\alpha_{N}$ has the effect of determining the prominence of the peaks of AMP $\left(\omega_{n}\right)$. The values of AMP $\left(\omega_{n}\right)$ for both systems are all prominent if $\alpha_{N}$ is small, but are greatly suppressed if $\alpha_{N}$ is large. Hence, $\alpha_{N}$ serves as a measure of the wave energy lost into the foundation due to its deformability.

\section{Effect of Layer Parameters on AMP $(\omega)$}

Four idealized layered systems were chosen to study the influence of layer parameters, such as the number of layers, layer stiffnesses, viscosities, etc., on the amplification spectrum. The first two systems are double-layered $(N=2)$. The third system is triple-layered $(N=3)$, and the fourth quadruple-layered $(N=4)$. The data for these systems are given in Table 1; they were chosen in such a way that the properties of the top layer and the foundation are the same for all of these systems. Also, the total thickness of the intermediate layers between the top layer and the foundation is 550 feet for all systems. The effect on AMP $(\omega)$ were studied by varying the number and the properties of the intermediate layers. In addition, to investigate the effect of material damping, in each layered system the amplification spectra for three different cases were calculated. These three cases were obtained by considering the layer media as

(1) nonviscous elastic solids,

(2) standard linear solids described by the parameters, $\tau_{j}$ and $r_{j}$, given in columns (5) and (6) respectively of Table 1 ,

(3) Voigt solids described by the same parameters, $\tau_{j}\left(r_{j}=\infty\right.$ in this case $)$.

The amplification spectra computed for each system are presented in Figures 4 (a) to 4 (d) respectively. Several observations can be made.

(a) The value of the fundamental characteristic frequency, $\omega_{1}$, is $5 \mathrm{rad} / \mathrm{sec}$ for the first system and is $7.2 \mathrm{rad} / \mathrm{sec}$ for the second system; whereas both the third and the fourth systems have a value of $\omega_{1}$ around $6.3 \mathrm{rad} / \mathrm{sec}$.

(b) The spectra for case (2) always lie between the spectra for case (1) and those for case (3).

(c) The spectra for the viscoelastic systems are less sensitive than those for the nonviscous systems to the variation of the parameters of the intermediate layers, and they appear very similar to each other for the cases $N=3$ and 4 . In addition, the spectra for the viscoelastic systems tend to decay with frequency, which is in agreement with theories showing that linear viscoelastic materials always attenuate wave amplitude according to a certain power of wave frequency.

Numerical calculations not shown here indicate that even if the number of intermediate layers is greater than 3 the general characteristics of AMP ( $\omega$ ) will not differ appreciably. Hence, it appears reasonable to conclude that, for a viscous multilayer system with $N \geqq 3$ and having layer stiffnesses increasing gradually with depth, if the properties of the top layer and the foundation are fixed, the general charac- 
teristics of AMP ( $\omega$ ) do not depend significantly on the properties of the intermediate layers so long as the total thickness of the intermediate layers remains unchanged.

(a) THE SINGLE - LAYER SYSTEM $(N=1)$

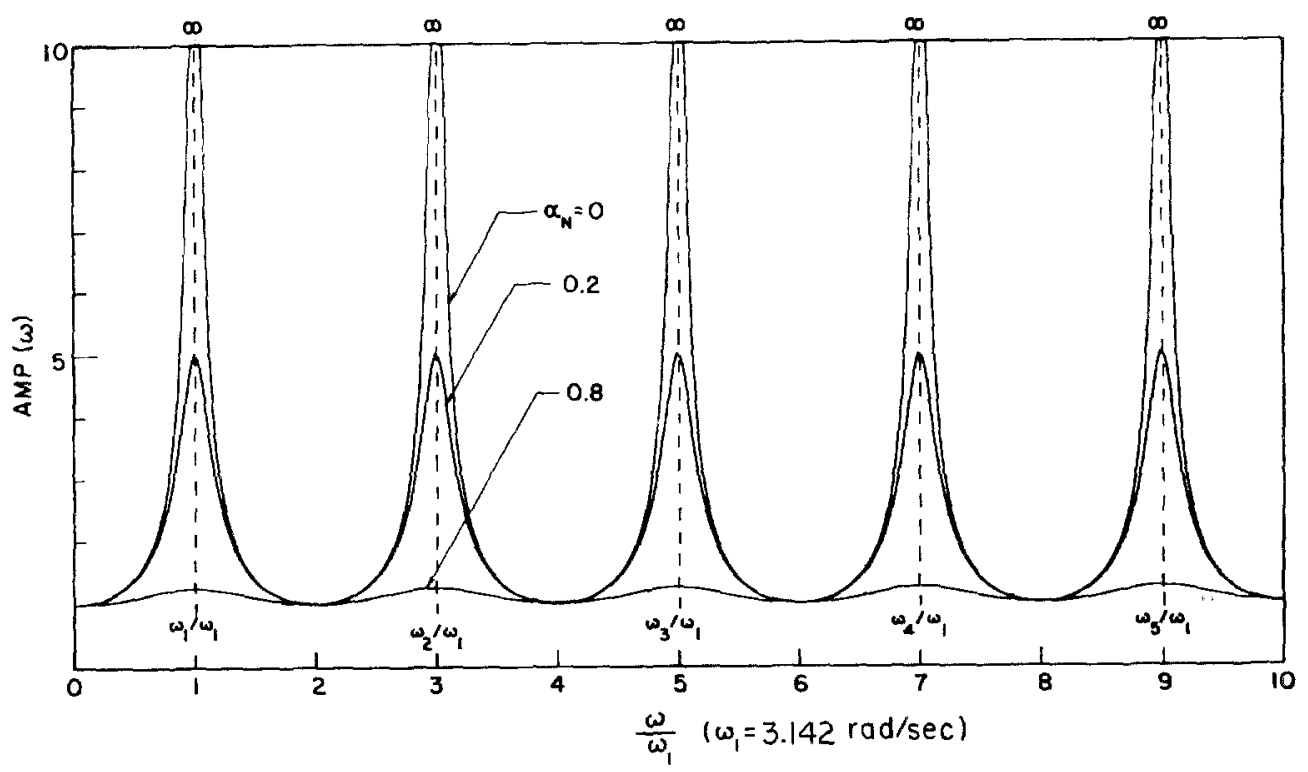

(b) THE TRIPLE- LAYER SYSTEM $(N=3)$

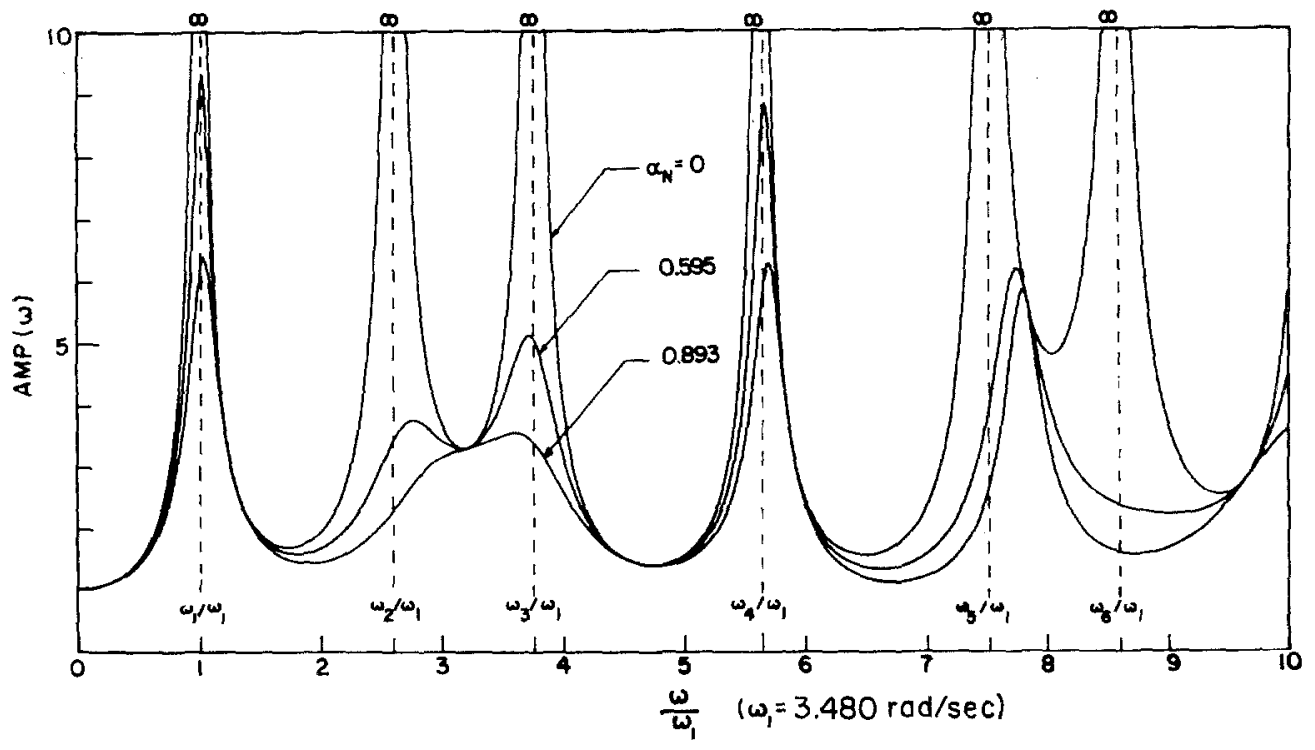

FIG. 3. Amplification spectra for different values of $\alpha_{N}$.

For practical problems involving viscous layered systems this implies that inaceuracy in measuring the properties of the intermediate layers will not introduce appreciable error in the computed surface motion. The above conclusion may not be valid for non- 
TABLE 1

Data for the Idealized Layer Systems

\begin{tabular}{|c|c|c|c|c|c|c|c|}
\hline System & $\begin{array}{c}(1) \\
\text { Layer No. } j\end{array}$ & $\begin{array}{c}(2) \\
\text { Layer Thick- } \\
\text { ness } H_{j}(\mathrm{ft})\end{array}$ & $\stackrel{(3)}{\operatorname{Density}} \rho_{(\mathrm{pfc})}$ & $\begin{array}{c}(4) \\
S \text { Wave Velocity } \\
c_{j} \text { (ft/sec) }\end{array}$ & $\underset{\tau_{j}(\mathrm{sec})}{\operatorname{Ri}(5)}$ & $\begin{array}{l}(6) \\
r_{j}\end{array}$ & $\begin{array}{l}(7) \\
\alpha_{j}\end{array}$ \\
\hline \multirow{4}{*}{1} & 1 & 200 & 110 & 1000 & 0.005 & 1 & 0.382 \\
\hline & 2 & 550 & 120 & 2400 & 0.002 & 5 & 0.240 \\
\hline & Base & & 150 & 8000 & 0 & 100 & \\
\hline & 1 & 200 & 110 & 1000 & 0.005 & 1 & 0.181 \\
\hline \multirow[t]{3}{*}{2} & 2 & 550 & 135 & 4500 & 0.001 & 20 & 0.506 \\
\hline & Base & & 150 & 8000 & 0 & 100 & \\
\hline & 1 & 200 & 110 & 1000 & 0.005 & 1 & 0.382 \\
\hline \multirow[t]{5}{*}{3} & 2 & 200 & 120 & 2400 & 0.002 & 5 & 0.474 \\
\hline & 3 & 350 & 135 & 4500 & 0.001 & 20 & 0.506 \\
\hline & Base & & 150 & 8000 & 0 & 100 & \\
\hline & 1 & 200 & 110 & 1000 & 0.005 & 1 & 0.385 \\
\hline & 2 & 150 & 120 & 2400 & 0.002 & 5 & 0.658 \\
\hline \multirow[t]{3}{*}{4} & 3 & 175 & 125 & 3500 & 0.0015 & 10 & 0.720 \\
\hline & 4 & 225 & 135 & 4500 & 0.001 & 20 & 0.506 \\
\hline & Base & & 150 & 8000 & 0. & 100 & \\
\hline
\end{tabular}
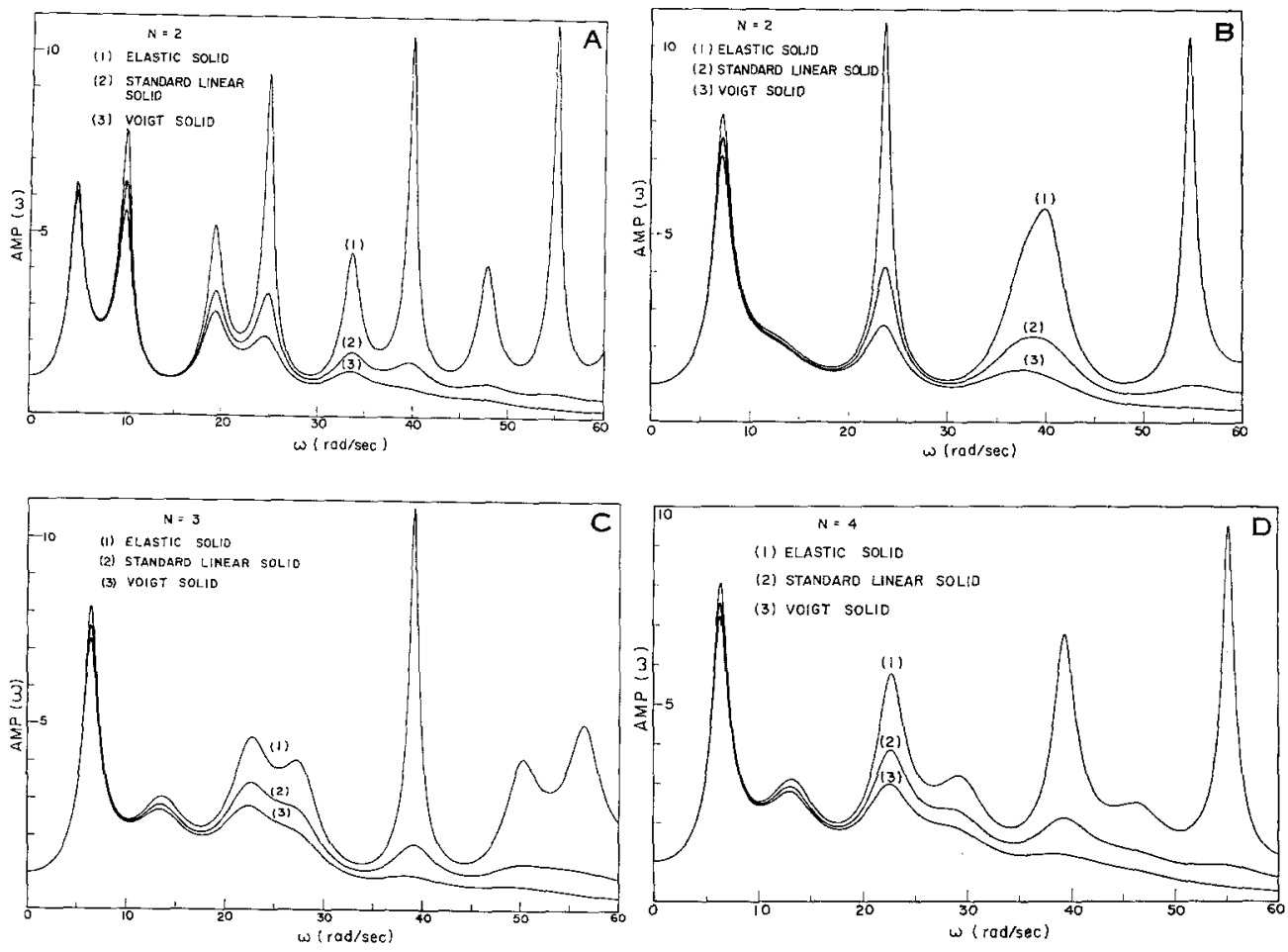

FIG. 4(a). Amplification spectra.

FIG. 4(b). Amplification spectra

FIG. 4(c). Amplification spectra.

FIG. 4(d). Amplification spectra.

viscous layered systems because they are more sensitive to the variation of layer parameters.

\section{Conclusions}

The steady-state response of an idealized layered system subjected to vertically incident sinusoidal waves was derived in the form of a recursion formula. The modi- 
fying effect of a layered system was expressed in terms of the amplification spectrum, AMP $(\omega)$. Four important properties were established regarding the nature of AMP $(\omega)$ of a nonviscous system having layer stiffnesses increasing with depth. From these properties, it was concluded that the impedance ratio $\alpha_{N}$ is a measure of the wave energy lost from the layers into the foundation due to its deformability, and determines the prominence of the resonant response of the system.

Numerical examples were given for studying the effect of layer parameters on the amplification spectrum. It was concluded that for a viscous multi-layer system with $N \geqq 3$ having stiffnesses gradually increasing with depth, the amplification spectrum depends primarily on the properties of the top layer and the impedance ratio between the bottom layer and the underlying half-space, and that the intermediate layers have small effect so long as their total thickness is fixed.

\section{ACKNOWLEDGMENTS}

The author is grateful to Professor G. W. Housner for providing advice and guidance. The research work was funded in part by the Engineering Division of the National Science Foundation.

\section{REFERENCES}

Haskell, N. A. (1953). The dispersion of surface waves in multi-layered media. Bull. Seism. Soc. Am. 43, 17-34.

Haskell, N. A. (1962). Crustal reflection of plane $P$ and $S V$ waves, $J$. Geoph. Res. 67, 4751-4767.

Herrera, I. and E. Rosenblueth (1965). Response spectra on stratified soil, Proc. 3rd World Conf. Earthq. Eng., Vol. 1, New Zealand.

Kanai, K. (1952), Relation between the nature of surface layer and the amplitudes of earthquake motions, I, Bull. Earthq. Res. Inst. 30, 31-37.

K ANAI, K. (1953). Relation between the nature of surface layer and the amplitudes of earthquake motions, II, Bull. Earthq. Res. Inst., 31, 219-226.

Kanai, K. and S. Yoshizama. (1953). Relation between the amplitude of earthquake motion and the nature of surface layer, III, Bull. Earthq. Res. Inst. 31, 275-279.

Kanai, K. and S. Yoshizama. (1956). Relation between the amplitude of earthquake motions and the nature of surface layer, IV, Bull. Earthq. Res. Inst. 34, 167-183.

Kanai, K. (1963). Some new problems of seismic vibrations of a structure, I, Bull. Earthq. Res. Inst. 53.

Kanai, K. (1964). Some new problems of seismic vibrations of a structure, II, Bull. Earthq. Res. Inst. 54 .

Matthiesen, R. B., C. M. Duke, D. J. Leeds and J. C. Fraser, (1964). Site characteristics of southern California strong-motion earthquake stations, Part Two, Rept. No. 64-15, Dept. of Eng., Univ. of Calif. Los Angeles.

Sezawa, K. and K. Kanai. (1930). Possibility of free oscillations of strata excited by seismic waves, Bull. Earthq. Res. Inst. 8, 1-11.

Sezawa, K. and K. Kanai. (1932). Possibility of free oscillations of strata excited by seismic waves, Bull. Earthq. Res. Inst. 10, 1-18 and 273-298.

Sezawa, K. and K. Kanai, K. (1935a). Decay constant of seismic vibrations of a surface layer, Bull. Earthq. Res. Inst. 13, 251-265.

Sezawa, K. and K. Kanai. (1935b). The rate of damping in seismic vibrations of a surface layer of varying density or elasticity, Bull. Earthq. Res. Inst. 13.

Takahasi, R. (1955). A short note on a graphical solution of the spectral response of the ground, Bull. Earthq. Res. Inst. 33, 259-264.

Tsai, N. C. (1969). Influence of local geology on earthquake ground motion, Ph.D. Thesis, California Institute of Technology, Pasadena.

Diviston of Engineming

California Institute of Technology

Pasadena, California

Manuscript received September 15, 1969. 